\title{
Reciprocal ST depression in acute myocardial infarction
}

\author{
OLUSOLA ODEMUYIWA, IAN PEART, CATHERINE ALBERS, ROGER HALL
}

From the Royal Victoria Infirmary, Newcastle upon Tyne

SUMMARY ST segment depression in leads remote from those showing ST elevation during acute myocardial infarction has been attributed to benign electrical phenomena, distant myocardial ischaemia, or extensive myocardial damage. Eighty four consecutive survivors under 55 years of age with a first transmural myocardial infarction were studied. All patients had exercise tests six weeks after infarction and coronary angiography a mean of three months after infarction. Thirty eight $(75 \%)$ of the 51 inferior and $19(58 \%)$ of the 33 anterior infarcts showed reciprocal ST depression of $\geqslant 1 \mathrm{~mm}$ during the acute phase. Ten $(26 \%)$ of the 38 patients with inferior infarcts and reciprocal depression had ST depression in the same leads on exercise. There was concomitant disease of the left anterior descending artery in four $(40 \%)$ of these 10 patients and in five $(18 \%)$ of the 28 with inferior infarcts with reciprocal depression but without ST depression in the same leads on exercise. Five $(26 \%)$ of the 19 patients with anterior infarcts with associated reciprocal depression and four of the 14 without reciprocal depression had important right coronary artery disease. In patients with inferior infarction important disease of the left anterior descending artery could not be predicted by ST depression in particular lead groups. Therefore reciprocal ST depression during acute myocardial infarction does not predict concomitant disease in the coronary artery supplying the reciprocal territory.

ST segment depression in leads remote from those showing ST segment elevation has been attributed to a wide variety of mechanisms. Some workers have suggested that this represents a benign electrical phenomenon. ${ }^{1-3}$ Others have suggested that it is due to ischaemia in another region distant from the infarct ${ }^{4-6}$ or that it is simply a manifestation of a large infarct. ${ }^{78}$

Jennings et al and Salcedo et al showed a correlation between anterior ST depression during acute inferior myocardial infarction and disease of the left anterior descending artery. ${ }^{69}$ Jennings et al also found ST depression in the same leads on exercise testing early after infarction, and this was interpreted as implying reversible ischaemia in the non-infarcted area. These studies, however, were of patients selected for angiography because of persistent symptoms or complications and they were probably biased towards the inclusion of patients

Requests for reprints to Dr R Hall, Royal Victoria Infirmary, Queen Victoria Road, Newcastle upon Tyne NE1 4LP.

Accepted for publication 16 July 1985 with extensive coronary artery disease. We have attempted to determine the relation, if any, between reciprocal ST segment depression in acute myocardial infarction and disease in the coronary artery supplying the reciprocal territory.

\section{Patients and methods}

This was a prospective study of 96 consecutive patients aged $\leqslant 55$ years admitted to the coronary care unit of this hospital with a first transmural infarction. Six patients died in hospital and six more were excluded because of heart failure (two), unwillingness to participate (two), or additional severe non-cardiac disease (two). The remaining 84 patients ( 74 males, 10 females; mean age 46.5 years) had exercise tests six weeks after infarction, and coronary angiography a mean of three months after infarction (range 1-6 months). The diagnosis of acute myocardial infarction was based on a history of prolonged chest pain, development of $Q$ waves, and a rise in the concentration of serum creatine kinase to at least twice that at baseline. Three 12 lead electrocardiograms (on admission and 24 hours and 48 
Table 1 Modified Bruce protocol used in study

\begin{tabular}{llll}
\hline Stage & $\begin{array}{l}\text { Duration } \\
(\text { min) }\end{array}$ & $\begin{array}{l}\text { Speed } \\
\text { (miles per hour) }\end{array}$ & $\begin{array}{l}\text { Incline } \\
\text { (degrees) }\end{array}$ \\
\hline 0 & 3 & $1 \cdot 2$ & 0 \\
$1 / 2$ & 3 & $1 \cdot 7$ & 5 \\
1 & 3 & $1 \cdot 7$ & 10 \\
2 & 3 & $2 \cdot 5$ & 12 \\
3 & 3 & $3 \cdot 4$ & 14 \\
4 & 3 & $4 \cdot 2$ & 16 \\
5 & 3 & 5.0 & 18 \\
\hline
\end{tabular}

1 mile per hour $=1.6 \mathrm{~km}$ per hour.

hours after the onset of the acute event) were recorded to identify reciprocal changes.

\section{ELECTROCARDIOGRAMS}

In inferior infarction ST depression in leads V1-V4, $\mathrm{I}$, and aVL was regarded as reciprocal. Reciprocal depression in these leads was further classified as ST depression in (a) V1-V4 alone; (b) I or aVL alone; or (c) those who had reciprocal depression in a combination of leads I, aVL, and V1-V4. In anterior infarction ST depression in any of leads II, III, or aVF was considered to be reciprocal. Reciprocal depression was defined as ST depression of $\geqslant 1 \mathrm{~mm}$ measured at least $0.08 \mathrm{~s}$ after the J point of the $\mathrm{QRS}$ complex in leads remote from the infarction site.

\section{EXERCISE TESTING}

Six weeks after infarction all 84 patients had exercise tests that followed a modified Bruce protocol (Table 1). Exercise started at Bruce level 0 and was stopped if the patient had severe symptoms, a drop in blood pressure of greater than $20 \mathrm{~mm} \mathrm{Hg}$, a run of three consecutive ventricular extrasystoles, or ST depression of $\geqslant 3 \mathrm{~mm}$.

\section{CORONARY ANGIOGRAPHY}

All patients had coronary angiography a mean of three months (range 1-6 months) after the acute infarction. All angiograms were reviewed by $\mathrm{RH}$ and IP and important stenosis was defined as $\geqslant 70 \%$ reduction in intraluminal diameter compared with the immediate proximal segment of normal coronary artery as measured in any cine projection. At least two orthogonal views of each coronary vessel were obtained. In anterior infarction the coronary artery supplying the reciprocal territory was the right coronary artery or a dominant circumflex artery and in inferior infarction the left anterior descending artery was regarded as supplying the reciprocal territory.

\section{STATISTICS}

The $\chi^{2}$ test and Student's unpaired $t$ test were used for statistical analysis.

\section{Results}

The site of infarction was inferior in $51(61 \%)$ and anterior in $33(39 \%)$ of patients.

\section{INFERIOR INFARCTION}

Reciprocal changes were seen on electrocardiograms from $38(75 \%)$ of the 51 patients with inferior myo-

Inferior site

\section{1 patients}

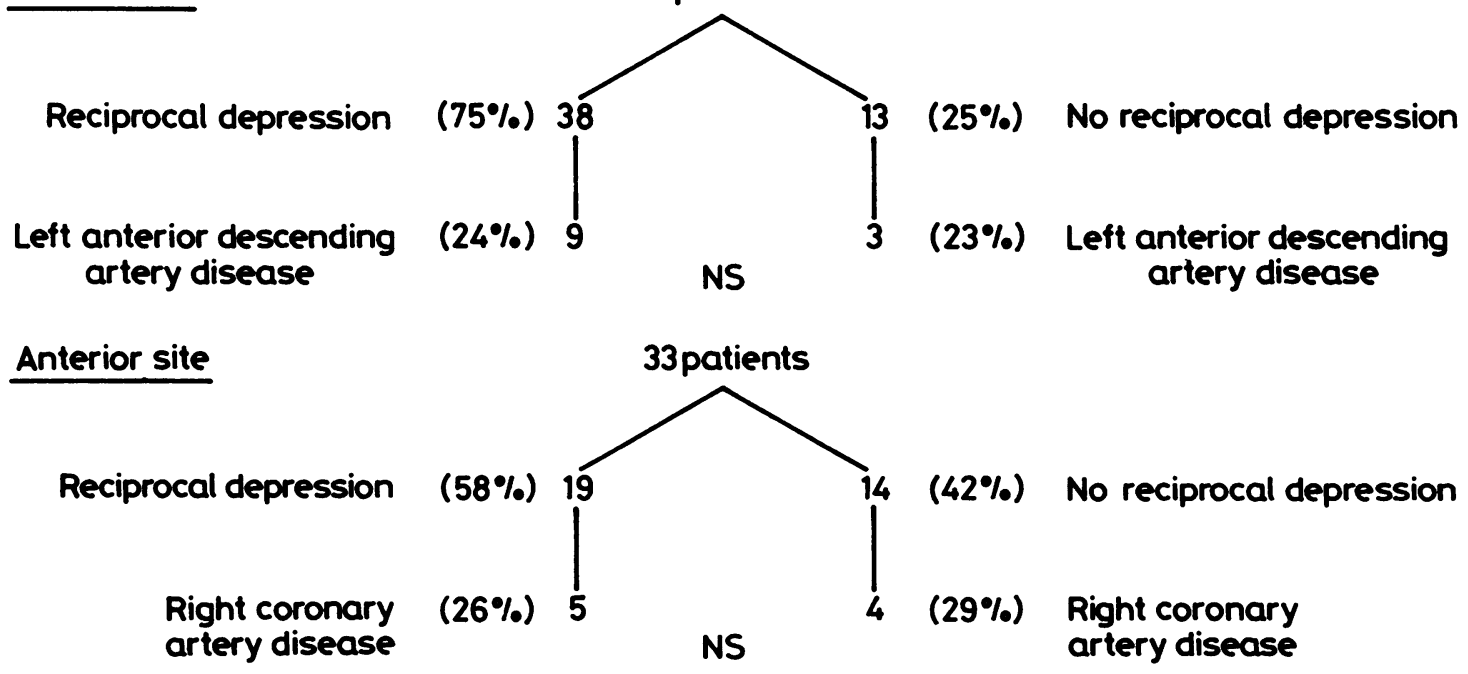

Fig. 1 Relation between reciprocal ST depression and important disease in artery supplying reciprocal territory. 
Table 2 Relation between $S T$ depression in different lead groups and disease in left anterior descending artery (LAD)

\begin{tabular}{llllll}
\hline Lead & Patients & LAD disease & Predictive & Sensitivity & Specificity \\
\hline V1-V4 & 4 & 0 & 0 & 0 & $88 \%$ \\
I, aVL & 12 & 2 & $17 \%$ & $22 \%$ & $74 \%$ \\
I, aVL, and V1-V4 & 22 & 7 & $32 \%$ & $78 \%$ & $66 \%$ \\
\hline
\end{tabular}

cardial infarction (Fig. 1). Nine (24\%) of the 38 patients showing reciprocal ST depression and three $(23 \%)$ of the 13 patients without reciprocal depression had important disease of the left anterior descending artery. Therefore the presence of left anterior descending artery disease was not predicted by reciprocal ST changes. Ten $(26 \%)$ of the 38 inferior infarct patients with reciprocal depression developed ST depression in the same leads on exercise (Fig. 2). Four ( $40 \%$ ) of these patients had important disease of the left anterior descending artery. Thus 28 patients showing reciprocal depression did not have ST depression in the same leads on exercise. There was important disease of the left anterior descending artery in five $(18 \%)$ of these 28 patients and four $(40 \%)$ of the 10 patients who had ST depression in the same leads on exercise. This difference was not statistically significant.

Table 2 shows the relation between ST segment depression in different lead groups and disease of the left anterior descending artery. Only seven $(27 \%)$ of 26 patients who showed ST depression in leads V1-V4 and nine $(26 \%)$ out of 34 patients with ST depression in leads I and aVL, had important disease of the left anterior descending artery. Therefore important disease of the left anterior descending artery could not be predicted by ST depression in any particular grouping of leads.

\section{ANTERIOR INFARCTION}

Nineteen $(58 \%)$ ) of the 33 patients with an anterior infarct showed reciprocal ST segment depression (Fig. 1). Five (26\%) of these 19 and four (29\%) of the 14 without reciprocal depression had severe right coronary artery disease. This difference was not statistically significant. Only one patient had ST depression in the same leads on exercise and this patient did not have severe right coronary artery disease (Fig. 2).

Inferior site

ST depression in same leads on exercise

Left anterior descending artery disease

Anterior site

\section{ST depression in same leads on exercise}

Right coronary artery disease

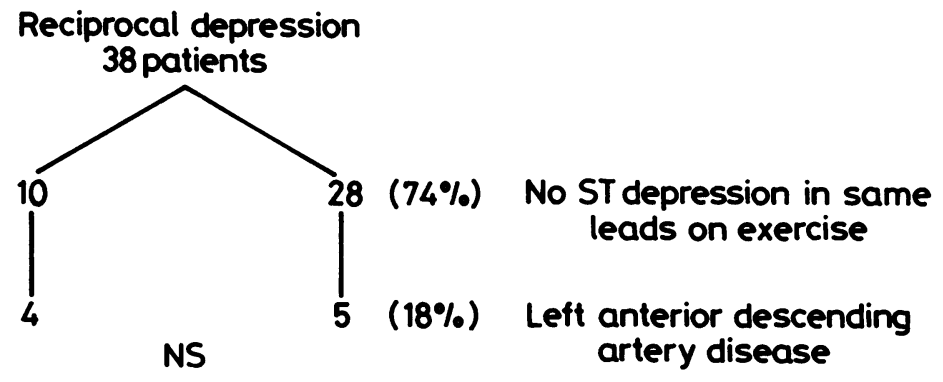

Reciprocal depression 19 patients

$(5 \%)$

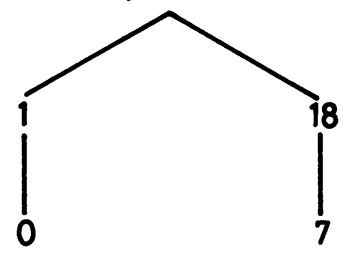

$(95 \%)$

No ST depression in same
leads on exercise

$(39 \%)$ Right coronary
artery disease

Fig. 2 Relation between acute reciprocal depression, $S T$ depression in same leads on exercise, and coronary artery disease in reciprocal territory. 
Table 3 Major complications occurring in hospital (complete atrioventricular block, left ventricular failure, supraventricular tachycardia, ventricular tachycardia)

\begin{tabular}{lll}
\hline & Patients & Complications \\
\hline Reciprocal depression & 57 & 13 \\
No reciprocal depression & 27 & 1 \\
Total & 84 & 14 \\
\hline
\end{tabular}

\section{COMPLICATIONS}

Three of the six patients who died in hospital had reciprocal depression and five of the six patients with inferior infarction who developed severe complications in hospital had reciprocal depression (Table 3). These included complete atrioventricular block, persistent angina, and ventricular and supraventricular tachycardia. All eight patients with anterior infarction who went on to develop severe complications in hospital had reciprocal ST depression. Therefore 13 out of 14 patients who developed severe complications during admission had reciprocal ST depression compared with 44 out of 70 patients who did not

Mean (SD) peak serum creatine kinase activity was 1900 (1350) units/1 in patients with reciprocal depression and 1500 (1265) units/1 in those without. This difference was not statistically significant.

\section{SYMPTOMS}

There was no significant difference between patients with and patients without reciprocal depression in the proportions who had angina at the time of coronary angiography.

\section{Discussion}

Reciprocal depression during acute myocardial infarction will have implications for management if patients with this feature constitute a high risk group in whom intervention to limit infarct size or alleviate distant ischaemia may favourably alter outcome. Wasserman et al found a correlation between the magnitude of inferior ST segment elevation and anterior ST segment depression in patients with inferior infarction. ${ }^{1}$ In a follow up for an average of more than three years they did not find any difference in mortality between those with and those without reciprocal precordial ST segment depression. They concluded that reciprocal ST segment depression during the early phases of inferior infarction was an electrical reflection of primary ST segment elevation in the area of infarction. Billadello et al used positron tomography to show that $67 \%$ of patients with reciprocal ST depression did not have anterior wall metabolic abnormalities during acute inferior infarction and they too concluded that reciprocal ST changes were electrical phenomena. ${ }^{10}$ They drew attention, however, to the finding that $33 \%$ of their patients had anterior wall metabolic abnormalities characteristic of ischaemia and suggested that in some cases reciprocal depression may represent distant ischaemia. Further support for this view came from Schuster and Bulkley in their review of 128 consecutive necropsies. ${ }^{4}$ They postulated that occlusion of one major coronary artery could interrupt collateral circulation and hence cause ischaemia in the distribution area of a second narrowed artery. Eight of their patients had angina after infarction and transient ST-T wave changes in the area supplied by a patent but critically narrowed vessel and they suggested that such patients may benefit from early aggressive intervention. Jennings et $a l$ and Salcedo et al lent further credence to this argument by showing a correlation between anterior segment ST depression during acute inferior myocardial infarction and disease of the left anterior descending artery. ${ }^{69}$ According to Salcedo et al reciprocal depression in leads V1-V4 was $95 \%$ predictive for disease in the left anterior descending artery. ${ }^{9}$ Jennings et al demonstrated ST depression in the reciprocal lead on exercise early after infarction in $63 \%$ of patients. ${ }^{6}$ They thought that this suggested important disease in the artery supplying this territory. In contrast in a large and detailed study Gibson et al showed that there was no significant difference either in the extent of underlying coronary artery disease or the frequency of left anterior descending artery disease between those with and those without reciprocal depression. ${ }^{7}$ Ferguson et al assessed angiographic results obtained during the acute phase of inferior infarction in six patients and also found that reciprocal depression did not predict the extent of coronary artery disease in the arteries supplying the remote wall. ${ }^{3}$

Only 10 out of 35 patients in the study of Jennings et al had coronary angiography. ${ }^{6}$ These 10 patients were catheterised because they had severe symptoms or complications. This could have biased the study towards patients with more extensive coronary artery disease. Our study consists of consecutive patients with acute anterior and inferior myocardial infarction who have had early routine postinfarction exercise tests and coronary angiograms without selection on the basis of symptoms. Our results accord with those of Gibson $e^{2} a^{7}$ and Ferguson et $\mathbf{l}^{33}$ but not with those of Jennings et $a l^{6}$ and Salcedo et al. ${ }^{9}$ We found that neither reciprocal depression nor exercise induced ST segment depression in the same leads early after infarction predicted concomitant 
disease in the coronary artery supplying the reciprocal territory. Unlike Salcedo et al we found that in acute inferior infarction reciprocal depression in specific lead groups V1-V4, I, and aVL does not predict concomitant disease in the left anterior descending artery.

Although several groups of workers, ${ }^{578}$ agree that reciprocal depression reflects more severe left ventricular damage, there are differences of opinions on what contribution remote wall damage makes to left ventricular dysfunction. Billadello $e t$ al found greater primary zone necrosis in those patients with reciprocal depression in acute inferior infarction than in those without reciprocal depression. ${ }^{10}$ Shah et $a l^{5}$ and Gibson $e t a l^{7}$ used non-invasive tests to show that reciprocal ST depression in acute inferior infarction was associated with greater global and regional left ventricular dysfunction. Goldberg et al obtained similar results. ${ }^{8}$ Shah et al suggested that these findings may be a reflection of non-transmural infarction of the remote anteroseptal wall, ${ }^{5}$ but Gibson $e t$ al believe that greater left ventricular dysfunction is simply a result of more extensive inferior or inferoposterior wall infarction and that anteroseptal wall ischaemia need not be invoked. ${ }^{7}$ Goldberg et al showed a correlation between reciprocal ST segment depression in acute inferior infarction and the extent of posterolateral wall damage, but they did not rule out anterior wall ischaemia as an additional factor that may contribute to left ventricular dysfunction.

Our study was not specifically designed to study the relation between reciprocal depression and infarct size but the higher frequency of complications in our patients showing reciprocal depression accords with the results of Gelman and Saltups. ${ }^{11}$ These findings suggest that patients with reciprocal depression have probably sustained larger infarcts.

Ian Peart was supported by the National Heart Research Fund and Catherine Albers by Bayer Pharmaceuticals.

\section{References}

1 Wasserman AG, Ross AM, Bogaty D, Richardson DW, Hutchinson RG, Rios JC. Anterior ST segment depression during acute inferior myocardial infarction: evidence for the reciprocal change theory. Am Heart $\mathcal{f}$ 1983; 106: 516-20.

2 Croft CH, Woodward W, Nicod P, et al. Clinical implications of anterior S-T segment depression in patients with acute inferior myocardial infarction. Am $\mathcal{F}$ Cardiol 1982; 50: 428-36.

3 Ferguson DW, Pandian N, Kioschos JM, Marcus ML, White CW. Angiographic evidence that reciprocal STsegment depression during acute myocardial infarction does not indicate remote ischemia: analysis of $23 \mathrm{pa}-$ tients. $A m$ 于 Cardiol 1984; 53: 55-62.

4 Schuster EH, Bulkley BH. Ischemia at a distance after acute myocardial infarction: a cause of early post infarction angina. Circulation 1980; 62: 509-15.

5 Shah PK, Pichler M, Berman DS, et al. Non-invasive identification of a high risk subset of patients with acute inferior myocardial infarction. Am $\mathcal{F}$ Cardiol 1980; 46: 915-21.

6 Jennings K, Reid DS, Julian DG. "Reciprocal" depression of the ST segment in acute myocardial infarction. $\mathrm{Br}$ Med F 1983; 287: 634-7.

7 Gibson RS, Crampton RS, Watson DD, et al. Precordial ST-segment depression during acute inferior myocardial infarction: clinical, scintigraphic and angiographic correlations. Circulation 1982; 66: 732-41.

8 Goldberg HL, Borer S, Jacobstein JG, Kluger J, Scheidt SS, Alonso DR. Anterior S-T segment depression in acute inferior myocardial infarction: indicator of posterolateral infarction. Am $\mathcal{F}$ Cardiol 1981; 48: 1009-15.

9 Salcedo JR, Baird MG, Chambers RJ, Beanlands DS. Significance of reciprocal $S-T$ segment depression in anterior precordial leads in acute myocardial infarction: concomitant left anterior descending coronary artery disease? Am f Cardiol 1981; 48: 1003-8.

10 Billadello J, Smith J, Ludbrook A, et al. Implications of "reciprocal" ST segment depression associated with acute myocardial infarction identified by positron tomography. 7 Am Coll Cardiol 1984; 3: 616-24.

11 Gelman JS, Saltups A. Precordial ST segment depression in patients with inferior myocardial infarction: clinical implications. Br Heart $\mathcal{f} 1982$; 48: 560-5. 